\title{
Hydronephrosis associated with pelvic organ prolapse: a review study
}

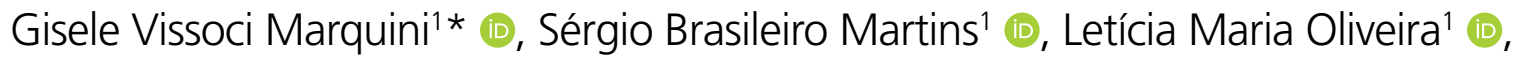 \\ Márcia Maria Dias ${ }^{1}$ (D), Claudia Cristina Takano ${ }^{1}$ (), Marair Gracio Ferreira Sartori ${ }^{1}$
}

\section{INTRODUCTION}

Pelvic organ prolapse (POP) is a common condition affecting more than 3 million women worldwide ${ }^{1}$. It may lead to bothersome pelvic pressure, bulge symptoms, and voiding dysfunction from bladder outlet obstruction, which may manifest as reduced flow rates and elevated postvoid residual volumes. Therefore, POP can also be associated with recurrent urinary tract infections, ureteral obstruction, and hydronephrosis $(\mathrm{HD})^{1,2}$.

The prevalence of HD in women with advanced POP has been reported to range from $3.6-30.6 \%$, with more severe prolapse associated with more HD. This condition is concerning because it can be completely asymptomatic. In addition, HD can result in silent kidney damage, which may lead to chronic or even end-stage renal failure ${ }^{1,2}$.

Despite the possible unfavorable outcome of advanced prolapse in renal function, there are currently no recommendations or guidelines regarding the evaluation of the upper tracts in patients with $\mathrm{POP}^{1}$. The majority of patients studied with POP experienced resolution of HD with treatment ${ }^{1}$.

The treatment of POP can be conservative or surgical. Pessary therapy is a conservative and practical option for patients with POP who were refusing surgery or being unsuitable candidates for surgery ${ }^{2}$. In cases with HD and renal failure, a simple pessary and transurethral catheter should be placed immediately to allow for complete bladder emptying ${ }^{2}$.

Faced with this background, the objectives of this review were to report the prevalence of POP associated with HD, to propose a practical diagnostic management, and to suggest immediate treatment options for POP on the resolution of HD.

\section{METHODS}

This study was conducted by a search of studies on Medline and PubMed using the terms "pelvic organ prolapse", "hydronephrosis", "pessary", and "pessary management". The Medline and PubMed searches identified 85 citations. First, all types of abstracts were selected (Books and Documents, Clinical Trials, Meta-Analysis, Randomized Controlled Trial, Review, and Systematic Review). Second, the abstracts were screened for the availability of the full text in English and 10 years publication date, resulting in the initial exclusion of 55 studies from the analysis.

Other 19 studies were excluded because they were case reports or they did not address the objectives of this review. A classic study (published in 1941) was included due to its relevance to describe the possible pathophysiological mechanisms associated with HD and POP. Therefore, 12 relevant studies were selected for this review. As this study was a review without access to patient documents, approval from the Institutional Review Board or Ethics Committee and informed consent were not needed.

\section{RESULTS}

Table 1 shows the relevant reviews and outcomes in the management of women with POP and HD according to evidence-based medicine.

Based on the most relevant and commonly management for HD associated with POP, the following didactic original algorithm with 10 steps is proposed immediately to diagnose and solve that association:

1. History and physical examination;

2. Diagnosis of POP: The Pelvic Organ Prolapse Quantification system (POP-Q) is clinically standardized and recommended;

\footnotetext{
'Universidade Federal de São Paulo, Departamento de Ginecologia, Setor de Uroginecologia e Cirurgia Vaginal - São Paulo (SP), Brazil.

*Corresponding author: giselemarquini@gmail.com

Conflicts of interest: the authors declare there are no conflicts of interest.

Received on May 01, 2021. Accepted on May 28, 2021.
} 
Table 1. Review of managing and treating HD in POP according to relevant evidence-based medicine.

\begin{tabular}{|c|c|c|c|c|}
\hline Author & Population or setting & Study design & Aims & Results (\%) \\
\hline $\begin{array}{l}\text { Dongol } \\
\text { et al. }{ }^{7}\end{array}$ & $\begin{array}{l}\text { Population of } 140 \\
\text { cases with POP who } \\
\text { underwent surgical } \\
\text { management. }\end{array}$ & Prospective study & $\begin{array}{c}\text { To establish the association } \\
\text { of renal impairment with } \\
\text { the degree and duration } \\
\text { of POP. }\end{array}$ & $\begin{array}{c}\text { Total } 5 \text { (3.57) patients had } \\
\text { HD. Total } 49 \text { (34.1) patients } \\
\text { had moderate-to-severe } \\
\text { renal failure. }\end{array}$ \\
\hline $\begin{array}{l}\text { Dancz } \\
\text { et al. }{ }^{10}\end{array}$ & $\begin{array}{l}\text { Population of } 180 \\
\text { participants with } \\
\text { advanced POP. }\end{array}$ & $\begin{array}{l}\text { Prospective study } \\
\text { on the prevalence } \\
\text { of HD in women } \\
\text { with advanced POP. }\end{array}$ & $\begin{array}{l}\text { To determine the } \\
\text { proportion of women } \\
\text { who demonstrate } \\
\text { improvement in HD after } \\
\text { pessary placement for } \\
\text { advanced POP. }\end{array}$ & $\begin{array}{l}\text { Successful pessary use } \\
\text { improved HD in over } \\
75 \% \text { of women with } \\
\text { advanced POP. }\end{array}$ \\
\hline Hui et al. ${ }^{12}$ & $\begin{array}{c}\text { Population of } 233 \\
\text { patients with POP } \\
\text { were followed } \\
\text { by sonographic } \\
\text { measurement of } \\
\text { bilateral renal pelvis to } \\
\text { identify the presence } \\
\text { of HD. }\end{array}$ & $\begin{array}{l}\text { Prospective } \\
\text { observational } \\
\text { study. }\end{array}$ & $\begin{array}{l}\text { To study the prevalence } \\
\text { of HD and its associated } \\
\text { factors in women with POP }\end{array}$ & $\begin{array}{l}\text { HD resolved in } 95 \% \text { of } \\
\text { patients after they received } \\
\text { treatment for POP. }\end{array}$ \\
\hline
\end{tabular}

HD: hydronephrosis; POP: pelvic organ prolapse.

3. Urinalysis and culture to investigate urinary tract infection;

4. Blood test: urea and creatinine to search renal insufficiency and blood count and culture to investigate possible sepsis;

5. Abdominal ultrasound (US) or abdominal computed tomography (CT) to identify the presence or absence of HD;

6. Treatment of POP: conservative option-Pessary. Pessaries are considered, according to this review, a wise choice to correct immediately the POP and minimize the deleterious effects of postrenal obstruction;

7. Drain bladder: spontaneously or with bladder catheterization;

8. If urosepsis: antibiotics;

9. Evaluate HD drainage (multidisciplinary team to provide, if necessary, nephrostomy);

10. Treatment of POP: surgical options can replace pessary treatment.

\section{DISCUSSION}

$\mathrm{HD}$ was more common in patients with advanced prolapse and among studies that used the POP-Q system (10.3-30.6\%) than Baden-Walker system (3.6-20.6\%) ${ }^{1}$. However, the prevalence can also vary by underdiagnosis, since this possibility of finding this association cannot be ruled out even in initial cases of prolapse ${ }^{1-3}$.
The mechanism by which POP causes HD is unclear; however, several theories have emerged. The pathophysiology is hypothesized to be somewhat different in patients with uterovaginal prolapse compared with those with vaginal vault prolapse. Multiple authors suggest that when the uterus is in situ, the genital hiatus entraps the ureters against the fundus of the uterus leading to ureteral compression ${ }^{1-3}$.

Others proposed that with procidentia, the uterine arteries cause ureteral compression by exerting downward traction on the ureters. Similarly, in patients with vaginal vault prolapse, the weakened cardinal ligaments could compress the ureters as the vaginal apex descends. Another possible mechanism could be obstruction at the level of the urethra causing backup of urine; however, this would not support the finding of a difference between uterovaginal and vaginal vault prolapse. Although the exact mechanism is unclear, additional discussion and possible upper tract imaging may be warranted in patients with severe uterovaginal prolapse ${ }^{1-3}$.

Clinical factors associated with HD include the degree of anterior or apical POP and diabetes mellitus ${ }^{4,5}$. In addition, the possible complications of the association of POP and HD, which can be a postrenal obstruction or renal impairment, are serious conditions, especially in elderly women, and require resolution of POP.

Recommendations for routine renal imaging for diagnostic purposes in patients with POP are widely disparate. HD can be assessed with renal US or CT imaging. Renal impairment can be assessed based on evaluations of serum urea and 
creatinine levels. If the increase in serum creatinine value is detected, possibly ureteral obstruction and renal insufficiency should be considered. If renal insufficiency is suspected, the US can be sufficient, particularly when the finding of bilateral HD is documented ${ }^{6}$.

Renal involvement linked to POP ranges from acute to chronic renal failure and may also lead to end-stage renal failure. Prolonged duration and the severity of POP are responsible for renal impairment. Dongol et al. reported among 140 cases of pelvic prolapse, a total of $3.57 \%$ of patients with HD. All 49 (34.1\%) patients had moderate-to-severe renal failure. And, 46 (32\%) patients in stage three showed moderate reduction in creatinine clearance, two $(1.4 \%)$ patients with severe reduction, and one $(0.7 \%)$ patient in end-stage renal failure ${ }^{7}$. Literature data show that untreated HD may progress to severe renal damage, suggesting that the resolution of HD results in either recovery or improvement of renal function ${ }^{1,7}$.

Both surgery and pessaries have shown a comparably positive effect on the symptoms of $\mathrm{POP}^{2}$, which suggests that there may be utility in performing a workup to investigate HD and correct POP. Therefore, if the HD is promptly diagnosed in patients with POP, the correction of POP, whether by pessary or surgery, can resolve $\mathrm{HD}^{2,8,9}$.

The use of a pessary to treat POP is a valid noninvasive option or conservative treatment and can solve in a conservative way the HD associated with POP. Some authors generally recommend a pessary trial for all new patients with POP. Many patients are surprised with the significant improvements they experience with a pessary trial ${ }^{8}$.

Furthermore, the use of a pessary can be a practical alternative to re-establish renal function in patients with $\mathrm{HD}$ awaiting surgery schedule for POP correction. An interventional trial, which aimed to evaluate the effect of the use of pessary on HD in women with advanced POP, determined an improvement in $\mathrm{HD}$ after pessary placement. According to the results, 75\% of women demonstrated an improvement in HD after pessary placement $^{9,10}$.

Despite the benefits of pessary use, there are some complications that are usually associated with neglected, oversized, or misplaced pessaries. Major complications may include fistulas, bowel or bladder erosion, and even $\mathrm{HD}^{6}$. In cases of pessary presence with a concomitant increase in serum creatinine value, a possible ureteral obstruction is suspected. In order to assess the presence of $\mathrm{HD}$ and its underlying causes, a CT scan should be performed to assess the mechanism of urinary tract obstruction. In case of renal insufficiency, abdominal US could be sufficient. If $\mathrm{HD}$ is detected in a patient with no signs of urosepsis, it is recommended a conservative management by the removal of the pessary and catheter placement. When urosepsis is suspected, it is mandatory to administer antibiotic therapy and evaluate the HD drainage by nephrostomy ${ }^{6}$.

Another option to approach HD and POP is surgical treatment ${ }^{9,11}$. In a retrospective case study on 250 patients presenting with severe uterovaginal prolapse, the authors evaluate the effect of the whole surgical correction of pelvic floor on HD due to severe prolapse ${ }^{8}$. As a result, HD was found in 32 (13.7\%) of 234 cases. According to that review, vaginal hysterectomy, axial vaginal apex suspension, and anterior and posterior repair result in either complete resolution or improvement of $\mathrm{HD}^{11}$.

In a prospective observational study, 233 patients with POP were staged by the POP-Q system, followed by abdominal US measurement of bilateral renal pelvis to identify the presence of HD. The follow-up scan for HD was performed after the patients were treated for the $\mathrm{POP}^{12}$. The prevalence of $\mathrm{HD}$ was $10.3 \%$ (95\% confidence interval [CI], 6-14\%). Although patient's age, higher parity, and the presence of diabetes mellitus and hypertension were more common in the group with $\mathrm{HD}$, the logistic regression analysis indicated that only the severity of POP was an independent risk factor for HD. The odds ratio in stages 3-4 POP for HD was 3.4 (95\% CI 1.3-9.2), and HD resolved in $95 \%$ of patients after receiving treatment for $\mathrm{POP}^{12}$.

A suggested follow-up, similar to that in conservative or surgical treatment, could be performed through an abdominal US during the diagnostic phase, before surgery, three days after surgery, and four weeks and six months after surgery. The HD cure criterion can be defined as no residual HD four weeks and six months after surgery ${ }^{11,12}$.

\section{Strengths and limitations}

There are currently few recommendations or guidelines regarding the evaluation of the upper tracts in patients with POP. This is a positive impact of the present review with a practical and objective approach to identify HD in women with POP. In addition, reviews such as this can stimulate the development of prospective studies with responses with a greater level of evidence on the importance of diagnosing renal complications in women with POP.

Another relevant aspect of this review is to value the association between POP and HD. Offering knowledge that POP correction can provide not only quality of life but also the recovery of a urinary system at risk of failure can make a difference in patient care.

The limitations of this study include the retrospective characteristics of the data collection, scarcity of data on this topic with no high-quality prospective or even randomized trials, and the lack of a long-term follow-up of the HD treatment in women with POP. These factors do not detract from the study because they should be the focus of a prospective trial. 


\section{CONCLUSIONS}

HD is not rare and is frequently found in patients with advanced POP. It is important for the medical team to be attentive and diagnose this possible renal complication in patients with POP. Most obstructive uropathy resolves with pessary use or surgical correction. For the future, prospective studies for this patient cohort should be planned.

\section{AUTHORS' CONTRIBUTIONS}

GVM: Data curation, Writing - review \& editing. SBM: Data curation, Writing - review \& editing. LMO: Data curation, Writing - review \& editing. MMD: Data curation, Writing - review \& editing. CCT: Data curation, Writing - review \& editing. MGFS: Conceptualization, Data curation, Writing review \& editing.

\section{REFERENCES}

1. Siddique $M$, Ingraham $C$, Kudish $B$, Iglesia $C B$, Polland $A$. Hydronephrosis associated with pelvic organ prolapse: a systematic review. Female Pelvic Med Reconstr Surg. 2020;26(3):212-8. https://doi.org/10.1097/SPV.0000000000000683

2. Farthmann J, Watermann D, Zamperoni H, Wolf C, Fink T, Gabriel B. Pelvic organ prolapse surgery in elderly patients. Arch Gynecol Obstet. 2017;295(6):1421-5. https://doi.org/10.1007/ s00404-017-4367-z

3. Lieberthal $F$, Frankenthal Junior $L$. The mechanism of ureteral obstruction in prolapse of the uterus. Surg Gynaecol Obstet. 1941;73:838-842.

4. Hussein NS, Rahman MN, Rifat UN. Bilateral pyonephrosis and end-stage renal disease secondary to pelvic organ prolapse. Saudi J Kidney Dis Transpl. 2013;24(4):810-2. https://doi. org/10.4103/1319-2442.113902

5. Dancz CE, Walker D, Thomas D, Özel B. Prevalence of hydronephrosis in women with advanced pelvic organ prolapse. Urology. 2015;86(2):250-4. https://doi.org/10.1016/j. urology.2015.05.005

6. Balzarro M, Rubilotta E, Porcaro AB, Trabacchin N, D'Amico A, Cerruto MA, et al. Original flow chart for the management of hydroureteronephrosis caused by pessary placement. Urologia. 2017;84(4):240-3. https://doi.org/10.5301/uj.5000266
7. Dongol A, Joshi KS, K C S. Renal impairment among patients with pelvic organ prolapse in a tertiary care center. Kathmandu Univ Med J (KUMJ). 2013;11(41):71-4. https://doi.org/10.3126/ kumj.v11i1.11031

8. Ahmed F, Sotelo T. Management of pelvic organ prolapse. Can J Urol. 2011;18(6):6050-3. PMID: 22166334

9. Thys SD, Roovers JP, Geomini PM, Bongers MY. Do patients prefer a pessary or surgery as primary treatment for pelvic organ prolapse. Gynecol Obstet Invest. 2012;74(1):6-12. https://doi.org/10.1159/000336634

10. Dancz CE, Walker D, Thomas D, Hussain N, Özel B. Effect of pessary use on hydronephrosis in women with advanced pelvic organ prolapse: a self-selected interventional trial. Int Urogynecol J. 2017;28(10):1589-93. https://doi.org/10.1007/ s00192-017-3289-4

11. Leanza V, Ciotta L, Vecchio R, Zanghì G, Maiorana A, Leanza G Hydronephrosis and utero-vaginal prolapse in postmenopausal women: management and treatment. G Chir. 2015;36(6):251-6. https://doi.org/10.11138/gchir/2015.36.6.251

12. Hui SY, Chan SC, Lam SY, Lau TK, Chung KH. A prospective study on the prevalence of hydronephrosis in women with pelvic organ prolapse and their outcomes after treatment. Int Urogynecol J. 2011;22(12):1529-34. https://doi.org/10.1007/s00192-011-1504-2 